\title{
MAINTENANCE OF NEURITE CONTACTS AT JUNCTIONAL REGIONS OF CULTURED INDIVIDUAL MUSCLE FIBERS FROM AGED RATS IS CORRELATED WITH THE PRESENCE OF A SYNAPSE-ASSOCIATED PROTEIN, GELASMIN
}

\author{
JEANNE C. JAY* and KATE F. BARALD** \\ Department of Anatomy and Cell Biology, University of Michigan Medical School, Ann Arbor, \\ Michigan 48109 (U.S.A.) \\ (Received September 7th, 1988) \\ (Revision received February 25th, 1989)
}

\section{SUMMARY}

When adult skeletal muscle is denervated as a result of injury or disease, it can usually be reinnervated [1-5]. Throughout the life of an animal, some skeletal muscles are thought to undergo cycles of denervation and reinnervation with concomitant remodelling of the neuromuscular junction, even in the absence of injury or disease [6-8]. In old animals, this reinnervation process may be faulty and may not occur at all in some aged muscle fibers, leaving them permanently denervated [6-8]. In general, the former endplate is the preferred site of reinnervation $[4,5,9]$, which has led to the speculation that specific molecular cues persist, particularly in the basal lamina of this region, that may mediate endplate reinnervation $[4,5,9,10]$. Although these molecular cues are as yet unidentified, one candidate is gelasmin, a $93 \mathrm{kD}$ glycoprotein we have isolated and characterized from preparations of rat synaptic extracellular matrix [11,12].

Because studies of reinnervation of aged muscle in vivo are extremely difficult to perform, we have devised a tissue culture model system of muscle reinnervation composed of isolated individual aged (17-26 months old) or young adult (3-5 months old; control) rat skeletal muscle fibers and embryonic (day 13 in utero) ventral spinal cord explants. We found that (1) gelasmin was present at all sites of nervemuscle contact on muscle fibers from both young adult and aged animals over a 10 day culture period, that (2) twice as many aged $(88 \%)$ as young adult fibers $(41 \%)$ had neurite contacts in the former junctional region at 10 days and (3) gelasmin was

*Present address: Dept. of Cell Biology and Anatomy, University of Alabama School of Medicine, Birmingham, Alabama, U.S.A.

**To whom all correspondence should be addressed. 
found on significantly more aged $(95 \%)$ than young adult fibers $(60 \%)$ grown without nerve explants. Furthermore, although no extrajunctional contacts were found on young adult fibers by the end of the 10-day culture period, substantial numbers of extrajunctional contacts were seen on aged fibers; gelasmin was present at all of these contact sites. These results are consistent with the idea that gelasmin, which is made by muscle fibers [12], may act to mediate or stabilize the contacts made by reinnervating nerve and that aged muscle fibers may regulate gelasmin or similar molecules differently from young adult muscle fibers.

Key words: Aged muscle; Culture; Spinal cord; Gelasmin

INTRODUCTION

We have been studying the molecular, cellular and functional properties of a synapse-enriched, basal lamina-associated $93 \mathrm{kD}$ glycoprotein called gelasmin $[11$ 13]. We have shown that gelasmin is distributed uniformly on embryonic rat muscle fibers before synaptogenesis and later is found co-localized with acetylcholine receptors $(\mathrm{AChR})$ on embryonic myotubes in culture in the absence of nerve contact $[11,12]$. Gelasmin is present at the endplate on all of the rat adult skeletal muscle fibers we have examined regardless of age [11-13]. We have also found that addition of purified gelasmin induces clustering of AChR on embryonic chick and rat myotubes in culture $[11,12]$ and that this clustering can be blocked with specific monoclonal antibodies we have made to gelasmin called SBL- 1 and SBL-2 $[11,12]$. Thus, gelasmin could be responsible for establishing and/or maintaining some aspects of synaptic organization.

We are presently testing the possibility that gelasmin may be a mediator of nervemuscle interactions during synaptogenesis and reinnervation. Because we have found that it is present at all sites of nerve-muscle contact both in vivo and in vitro, as well as at former endplates in the absence of nerve [11-13], gelasmin or a similar molecule could provide cues for reinnervating nerves as they interact with denervated muscle fibers.

At least some aged muscle fibers may undergo repeated cycles of degeneration and regeneration [6-8] in the normal course of development and ageing, and some aged muscle fibers become functionally permanently denervated as a result of faulty reinnervation processes [6-8]. However, there is a great deal of variation in the response of different types of muscle fibers to the ageing environment $[6-8,14-18]$ which may be due, at least in part, to the amount of use the muscle receives $[6,7]$ For example, pronounced changes, including increasing incidence of denervation and neuromuscular junction remodelling have been reported in the soleus muscle $[6,7]$, a muscle that is not constantly used in ageing animals. However, neuromuscular junctions of the diaphragm which is in constant use throughout life. 
have been reported to undergo little change during ageing [6]. Increases in the length of primary synaptic clefts in aged mouse extensor digitorum longus muscle (EDL) [18] have been postulated to be compensatory for loss of girth in the junction, so that function remains unimpaired.

We wanted to explore the possibility that withdrawal of nerve contact from aged fibers and failure to reform such contacts on aged denervated fibers reflected the loss of potential molecular cues for reinnervation such as gelasmin (or similar molecules). However, studies of denervation and reinnervation of aged skeletal muscle at the molecular level in vivo are complicated by the increased incidence of disease among old animals and the increased amount of connective tissue and fat that accumulate among skeletal muscle fibers [3]. The process of nerve-muscle interaction cannot be monitored over its entire time course and it is difficult to manipulate the experimental system in vivo.

We therefore developed a nerve-muscle tissue culture system that lends itself easily to experimental manipulation and observation [19]. Single aged individual flexor digitorum brevis (FDB) skeletal muscle fibers from rats 17-26 months old or those from young adult control animals 3-5 months old were cultured with or without embryonic rat ventral spinal cord explants (from embryos 13 days in utero) as a source of nerve.

The most important finding of this study was that, although gelasmin was present at all neurite contact sites on both aged and young adult muscle, twice as many aged fibers $(91 \%)$ as young adult fibers $(45 \%)$ retained gelasmin at the former junction during the culture period. We also found that the aged FDB individual muscle fibers in culture had larger numbers of neurite contacts in both junctional and extrajunctional regions after 10 days in culture than young adult fibers, and all sites, including extrajunctional sites were found to have gelasmin. These results are consistent with the hypothesis that gelasmin, or some similar molecule, might serve either as a target molecule for reinnervating nerve or may serve to stabilize nerve-muscle interactions at regenerated endplates.

\section{MATERIALS AND METHODS}

\section{Isolation and culture of individual adult skeletal muscle fibers}

Young adult muscle fibers. Cultures of young adult muscle fibers from 3-5 months old rats were prepared as previously described [11-13]. Fibers from 40 animals were used in these experiments. The collagenase dissociation method was developed by Bekoff and Betz [20]. We have modified the technique to produce cultures with enough viable fibers for quantitative analysis in a variety of experimental paradigms [11-13]. One FDB muscle was dissected from each foot of an 80300 g Sprague-Dawley rat. Dulbecco's phosphate buffered saline (D-PBS) (GIBCO) supplemented with final concentrations of $1.8 \mathrm{mM} \mathrm{Ca}^{++}, 0.32 \mathrm{mM} \mathrm{Mg} \mathrm{M}^{++}$ and $6 \mathrm{mM}$ glucose was used as a bathing medium while fascia were removed. 
Muscles were then transferred to a solution of collagenase (Sigma, Type I) that had 600 units of collagenase activity/ml Dulbecco's PBS (D-PBS; GIBCO formulation). Dissociation proceeded for $1.5 \mathrm{~h}$ on a slow speed rotator (Cole-Parmer Instrument Co., Chicago, IL) at $37^{\circ} \mathrm{C}$. Muscles were then placed in the following nutrient medium: Dulbecco's Modified Eagles Medium (D-MEM) (GIBCO) supplemented with $10 \%$ horse serum (GIBCO) that was heat inactivated for $60 \mathrm{~min}$ at $56^{\circ} \mathrm{C}$ and spun for $1 \mathrm{~h}$ at $4000 \mathrm{~g}, 0.29 \mathrm{~g} / \mathrm{l} \mathrm{L}$-glutamine (GIBCO), $1 \%$ antibiotic-antimycotic (penicillin-streptomycin-fungizone mixture from M.A. Bioproducts), and $0.5 \%$ garamycin (M.A. Bioproducts). Tetrodotoxin (TTX) (Sigma) was added to some cultures in a final concentration of $10^{-7} \mathrm{M}$ to prevent excessive muscle contraction Fiber bundles were gently teased from tendons and as much connective tissuc as possible removed. These muscle fiber bundles were then subjected to further dissoci ation in a $1: 1$ ( $\mathrm{vol} / \mathrm{vol}$ ) dilution of the collagenase solution (as described above) with nutrient medium for $30 \mathrm{~min}$ on the slow speed rotator at $37^{\circ} \mathrm{C}$.

Single fibers were then collected, suspended in a Vitrogen (Flow Labs) gel mixture consisting of $1 \mathrm{ml}$ Vitrogen, $0.125 \mathrm{ml} 5 \times$ D-MEM, $0.05 \mathrm{ml} 0.14 \mathrm{M} \mathrm{NaOH}$ and 0.04 $\mathrm{ml} 0.9 \mathrm{M}(7.5 \%) \mathrm{NaHCO}_{2}$. In order to provide the best optical conditions for fluorescence microscopy or Smith interference contrast microscopy, $35 \mathrm{~mm}$ tissue culture dish bottoms (Falcon) were replaced with glass cover slips glued in place with Sylgard (Dow Corning) [19].

Cultures were incubated at $37^{\circ} \mathrm{C}$ for $20 \mathrm{~min}$ in $95 \% \mathrm{O}$, and $5 \% \mathrm{CO}$, to allow the Vitrogen mixture to gel; subsequently, $1 \mathrm{ml}$ of enriched medium, which consisted of nutrient medium plus the addition of $5 \%$ chicken embryo extract $[19]$ or $10 \mu \mathrm{g} / \mathrm{m}$ ! ovotransferrin (conalbumin, Sigma) [19] was placed in each dish and changed every other day.

Aged fibers. The FDB from 37, 17-26 months old, Sprague-Dawley rats wa: used as a source of aged muscle fibers. The dissociation protocol and culture preparation were essentially those described above.

Dissociation of two aged FDB muscles yielded an average of eight individual dishes containing 130-150 fibers per dish. In some experiments as tew as 50 fiber. or as many as $\mathbf{3 0 0}$ fibers/dish were plated.

\section{Muscle fiber viability}

Prior to the application of other histochemical stains, $0.1 \%$ trypan blue was used in cultures to determine muscle fiber viability, as previously described $[11,12,19]$. This dye was not incorporated by viable cells, whereas non-viable cells took up the dye $[21,22]$. In some experiments muscle fiber viability was determined by applying $1 \mathrm{part} / \mathrm{million}$ each acridine orange and ethidium bromide (Sigma) in PBS [23]; live cells contained green nuclei and dead cells orange nuclei when viewed with an epifluorescence microscope equipped with FlTC optics [23]. Phase-contrast criteria. including the presence of definite cross-striations and peripherally located nuclei, were also used to confirm fiber viability. 
Only those fibers that remained viable during the 10-day culture period in cultures of muscle alone and in muscle-spinal cord explant cultures (nerve-muscle cultures; $n$-m cultures) were used for data collection. Fibers were observed at 24-h intervals during the culture period.

\section{Spinal cord explants}

Spinal cord explants were prepared by our previous methods [19]. Embryonic rat pups on gestational days 12-14 were removed from the uterine horns of a gravid female rat that was killed by brief ( $1-2 \mathrm{~min})$ exposure to $\mathrm{CO}_{2}$. Embryos were killed by severing the neck, and the cords removed whole after cutting open the vertebrae. The spinal cords were divided into dorsal and ventral portions along the entire length. The dorsal halves of the cord were discarded and the ventral halves were cut into $0.1-0.5 \mathrm{~mm}^{2}$ segments which were then placed in Vitrogen gels for "initial outgrowth" in nutrient medium (see above). After $24 \mathrm{~h}$, the initial outgrowth of neurites was excised and the ventral explant cores placed in muscle fiber cultures as described below, or dissociated for single cell culture as described below.

When we designate nerve-muscle cultures as "co-cultures" it means that the muscle fibers were cultured with spinal cord ventral explants. Not every muscle fiber in the co-culture is contacted by neurites; however, the muscle fibers that were followed over time in co-cultures had all been contacted by neurites.

\section{Nerve-muscle co-cultures}

Ventral spinal cord explant cores were removed from the Vitrogen gel (see above) and positioned in target fields of 1-6 muscle fibers in 1-day-old muscle cultures. The distance between explants and muscle fibers was approximately $0.1-0.7 \mathrm{~mm}$. Each explant was secured with a drop of Vitrogen mixture (see above). Nutrient medium $(1 \mathrm{ml})$ was then placed in each dish and the medium changed every other day.

\section{Dissociated embryonic rat spinal cord cultures}

Dissociated rat embryonic spinal cord cultures were prepared by our previously described method [24]. Briefly, the ventral spinal cord segments (see above) were treated with $0.1 \%$ trypsin (Gibco) in Modified Puck's glucose containing calcium and magnesium at $37^{\circ} \mathrm{C}$ for $11 \mathrm{~min}$ [24]. The reaction was then stopped by the addition of nutrient medium containing serum (see above.) Dissociated cells were centrifuged in a clinical table-top centrifuge and resuspended in the same growth medium that was used to grow the muscle fiber cultures (see above).

Cells were plated on collagen substrates [19] or in collagen gels [19] alone or in the presence or absence of specific numbers of young adult or aged muscle fibers in groups containing 50 fibers ( \pm 10 fibers), 100 fibers ( \pm 18 fibers) and 300 fibers ( \pm 27 fibers). Nutrient medium was changed every other day. These cultures were used to assess the effect of the presence of aged or young adult muscle fibers on the survival of embryonic neurons. 
Neuronal cell numbers were determined by counting the number of individual neuronal cell bodies with time in 25 random fields at $320 \times$ under a Leitz phase contrast microscope on a daily basis over the 10-day period.

\section{Localization of acetylcholine receptors and acetylcholinesterase on muscle fibers}

In order to observe putative AChRs, defined as rhodamine bungarotoxin ( $R$ BTX) binding sites, cultures were incubated for $1 \mathrm{~h}$ at room temperature or $30 \mathrm{~min}$ at $37^{\circ} \mathrm{C}$ with $1 \times 10^{-8} \mathrm{M}$ R-BTX [25]. Cultures were subsequently rinsed with PBS $-1 \%$ BSA followed by PBS. All accessible, fully intact, viable muscle fibers in the dish were examined for R-BTX binding sites, with the use of an oil immersion $(50 \times)$ objective, on a Leitz Diavert microscope that had appropriate narrow-band filter combinations for tetramethylrhodamine isothiocyanate (TRITC) or fluorescein isothiocyanate (FITC) (see below). Kodak Ektachrome 400 film was used for photography; the ASA was set at 200 with a Leitz Vario Orthomat.

If $\mathrm{AChE}$ was assayed, cultures were fixed for $30 \mathrm{~min}$ prior to examination of $\mathrm{AChR}$ in a Liebowitz-15 (L-15) medium (GIBCO) to which $0.15 \%$ glutaraldehyde and $2 \%$ formaldehyde were added [26]. Cultures were then rinsed with phosphate buffered saline (PBS) (see below) and then with PBS containing 1\% bovine serum albumin (BSA). Cultures were then incubated in a histochemical stain for acetylcholinesterase (AChE) [26]. The staining solution was removed after $1-5 \mathrm{~h}$ and cul tures were rinsed with PBS. Labeled fibers were then relocated by means of an ocular micrometer grid, and their stage micrometer coordinates, reanalyzed for $\mathrm{AChE}$, and photographed again. An inhibitor of "true" AChE, Burroughs Well come anti-cholinesterase BW284C51 $\left(10^{-7} \mathrm{M}\right)$ and an inhibitor of non-specific esterase, iso-OMPA $\left(10^{-6} \mathrm{M}\right)$ (Koch-Light, Beckenham, England) were used to confirm that the esterase at the junction was, in fact, true AChE.

Confirmation of the presence of junctional $A C h E$ and $A C h R s$ was made on samples of muscle taken prior to dissociation and on $0 \mathrm{~h}$ cultures of muscle (muscle fibers examined immediately alter dissociation and plating). Fibers teased from fresh muscle were placed on a $45 \times 60 \mathrm{~mm}$ cover slip. After the medium was removed, fibers were stained either with R-BTX to visualize AChRs or with a modified Karnovsky stain that revealed AChE [26] or by both together. PBS contained $2.7 \mathrm{mM} \mathrm{KCl}, 1.2 \mathrm{mM} \mathrm{KH}_{2} \mathrm{PO}_{4}, 137 \mathrm{mM} \mathrm{NaCl}, 8.1 \mathrm{mM} \mathrm{Na}_{2} \mathrm{HPO}_{4} 7 \mathrm{H}_{2} \mathrm{O}$. $0.72 \mathrm{mM} \mathrm{MgSO}_{4}, 0.16 \mathrm{mM} \mathrm{CaCl}_{2}$ and $50 \mathrm{mM}$ glucose. In this study, only qualitative measurements of the synaptic molecules were made; no attempt was made to determine relative densities or quantitation of either AChR or AChE.

\section{Preparation of $R-B T X$}

Rhodamine was conjugated to alpha-bungarotoxin according to the procedure described by Ravdin and Axelrod [25]. Rhodamine B isothiocyanate was obtained from Sigma. Alpha-bungarotoxin was obtained from Biotoxins, Inc., St. Cloud, FL. 


\section{Identification of former endplate regions}

At the beginning of an experiment, each culture dish containing muscle fibers was marked with three narrow scratches incised on the sides with a diamond pencil in asymmetrical locations (e.g. at $90^{\circ}, 140^{\circ}$ and $210^{\circ}$ ). These marks corresponded to marks drawn on the moveable culture dish holder mounted on the stage of the Leitz inverted microscope so that each dish could be placed in the same position for viewing on subsequent days in culture. All dishes in a given experiment were identically marked. Location of each muscle fiber in the dish was noted by making a record of the stage micrometer coordinates and by making a photographic record and/or camera lucida tracing of each fiber with its associated landmarks (endplate location, contour, etc.). We had no difficulty in identifying the endplate region of living fibers on day 1 in culture: it was photographed using both low $(10 \times)$ and high power $(32 \times)$ objectives and/or a camera lucida tracing was made while noting its exact location with the stage micrometers.

Endplate sizes and shapes at day 1 were identified by R-BTX staining; we measured the broadest dimension of the endplate with an ocular micrometer. We could relocate these former endplate regions during the subsequent 10-day period, both because the stage micrometer coordinates were known and because one or more of the histochemical stains (for AChE or AChR; see above) or immunohistochemical stains for gelasmin (see below) could be used to relocate the endplate during this time. Morphological criteria assessed by Smith interference contrast microscopy were also used to confirm the viable state of the fibers. These criteria included the presence of junctional folds and clusters of 6-12 subjunctional nuclei (see Figs. 1 and 2). In the rare case that all of these molecular and histochemical markers were lost from the endplate, however, we found that the former endplate region could be found by using the micrometer coordinates. The fibers underwent very little or no morphological change in the Vitrogen gels during this period as long as they remained viable; dead fibers were automatically eliminated from the observations. Fibers had to survive the entire culture period to be counted, and there was little change in viability over the culture period (see results) (Fig. 3).

\section{Determination of frequency of neurite association with junctional and extrajunctional regions}

With Smith interference contrast optics, the number of neurites associated with junctional and extrajunctional regions was determined on muscle fibers on a daily basis during the culture period. Muscle fibers were selected in which the junctional region could be identified on day 1 in culture by two (or more) of three molecular criteria: gelasmin localization, AChR localization as well as AChE localization (if the experiment was terminated after $\mathrm{AChE}$ staining). Confirming morphological specializations junctional folds, clusters of $6-12$ subjunctional nuclei) were also noted. Fibers were included for data collection provided they were not contracted in the junctional or adjacent extrajunctional regions (see above and Fig. 1). 
The exact location of each endplate was noted using stage micrometer coordinates and camera lucida and/or photographic recording of the muscle fiber An ocular micrometer with a $1 \mathrm{~mm}^{2}$ grid, which was subdivided into $0.1 \mathrm{~mm}^{2}$ units was used to determine the number of neurite contacts along the entire length of the muscle fiber.

Fiber lengths of both young adult and aged muscle fibers ranged from $1.1102 \mathrm{t}$ $\mathrm{mm}$. The distance from the center of the explant to the center of the muscle fiber was also measured. Comparisons cited are among fibers within $20^{\circ}$ of the perpendicular to the main vector of the centrally located explant. Fibers that were more than $20^{\circ}$ from the perpendicular were not counted. Neurite outgrowth was profuse over the $360^{\circ}$ circumference of the explant and some neurites were as long as $2-3 \mathrm{~mm}$.

Neurite contacts in junctional or extrajunctional "units" of the grid on the muscle fibers were counted by first localizing the endplate region by molecular, morphological and/or the micrometer coordinates identified on day 1 . The position of the fibers in the Vitrogen gel did not change over the 10-day culture period, it: long as the fiber remained viable; therefore the position of the original endplate als did not change. Thus, the original endplate region could be recognized even after the loss of morphological and/or molecular junctional landmarks, although the loss of all such markers was extremely rare.

In order to count the number of neurite contacts in former junctional regions, the microscope was initially focused on the plane of the upper surface of the muscle fiber; the number of neurites within the $1 \mu \mathrm{m}^{2}$ area was recorded. Focusing and neurite counting were continued until the lower surface of the muscle fiber wa, reached. Total distance between upper and lower fiber surfaces ranged from $45-60$ $\mu \mathrm{m}$. Neurites assigned to the synapse itself in the junctional (EP) region were those in the focal plane of the endplate; endplates essentially filled the $0.1 \mathrm{~mm}^{2}$ unit. We did not count the neurites that touched the muscle fiber surface at other optical planes in the junctional "segment" outside the synaptic region itself as junctional

\section{Gelasmin}

The presence of the synapse-enriched protein, gelasmin, a $93 \mathrm{kD}$ protein was monitored with a gelasmin-specific monoclonal antibody, SBL-2 [12]. This molecule had previously been isolated from adult rat diaphragm $[11,12]$ and characterized in adult rat diaphragm in vivo $[11,12]$ and embryonic rat muscle myotube cultures [12] SBL-2 is a mouse IgG monoclonal antibody that was affinity purified from mouse ascites fluid as described in [12]. An FITC conjugated second-step rabbit anti-mousc IgG antibody was used to visualize the SBL-2 antibody binding sites.

Monoclonal antibody SBL-2 is capable of identifying gelasmin on Western blot [12] as well as at the neuromuscular junctions of embryonic and adult muscle fibers both in vitro and in sectioned material obtained from whole muscles [12]. Neither the SBL-2 antibody [12] nor another antibody, SBL-1 [11,12], which we made to components of the isolated adult rat diaphragm synaptic extracellular matrix [11] 
and which recognizes the same $93 \mathrm{kD}$ protein, recognizes any epitopes in embryonic or adult nerve tissue $[11,12]$. Controls for antibody staining of the tissue cultured adult muscle fibers included omission of the primary antibody and substitution of a non-specific monoclonal antibody (to chick neural crest cells) for the primary antibody.

\section{Statistics}

The Kruskal-Wallace (K-sample) [27] test for non-parametric number distribution, $P<0.05$ was used to determine the statistical significance of changes in AChE, AChR, number of neurite contacts, neuronal survival, muscle fiber survival and gelasmin distribution on muscle fibers cultured alone or muscle fibers in nervemuscle cultures.

\section{RESULTS}

Are the properties of aged muscle fibers in vitro similar to those in vivo?

If our tissue culture system is to provide a good model for reinnervation of aged muscle fibers, the properties of the aged cultured muscle fibers must approximate those seen in the living animal. This was the case for the FDB muscle. The FDB muscle was chosen by us and by others $[13,19,20,28]$ for studies in culture because it is a palmate muscle with fibers which are short and easy to dissociate individually (Fig. 1). This is not true of muscles with longer fibers such as the rat soleus, tibialis anterior or EDL, which are not amenable to culture. When we compared the properties of the FDB in vivo and in vitro we found that, for example, the diameter of aged cultured $(72 \mu \mathrm{m} \pm 4.9, n=50)$ and freshly prepared $(75 \mu \mathrm{m} \pm 6.1, n=37)$ aged muscle was the same. All measurements in this paper are expressed as: (mean \pm S.E.M. and $n=$ the number of replications). However, the diameter of young adult fibers in culture $(50 \mu \mathrm{m} \pm 2.9, n=44)$ was significantly smaller. The average length of aged muscle fibers in our cultures, $(1.4 \mathrm{~mm} \pm 0.20, n=57)$ was not significantly different from that of young adult muscle fibers $(1.7 \mathrm{~mm} \pm 0.1, n=$ $68)$, which does not differ from that of freshly prepared young adult fibers $(1.8 \mathrm{~mm}$ $\pm 0.2, n=130)$ or freshly prepared aged muscle fibers $(1.6 \mathrm{~mm} \pm 0.2, n=120)$. At 26 months in vivo we found no evidence of fiber atrophy in FDB muscle fibers. Thus, the aged cultured fibers are morphologically similar to those in aged animals.

Sizes of intact endplates were also compared on aged fibers in culture at early times after plating (Fig. 2) and in vivo. Most endplates are either eliptical or roughly circular. If the endplate was eliptical in shape, the long axis of the elipse is given as the "diameter". The diameter of endplates on aged muscle fibers in vitro $(57 \mu \mathrm{m} \pm$ $2.3, n=31)$ was the same as that in vivo $(61 \mu \mathrm{m} \pm 4.3, n=22)$ but was significantly larger than that of young adult muscle fibers $(33 \mu \mathrm{m} \pm 5.1, n=35)$ in vitro. Endplates on aged cultured muscle fibers also had more elaborate junctional folds than did endplates on young adult muscle fibers (Fig. 2) as has been reported for 


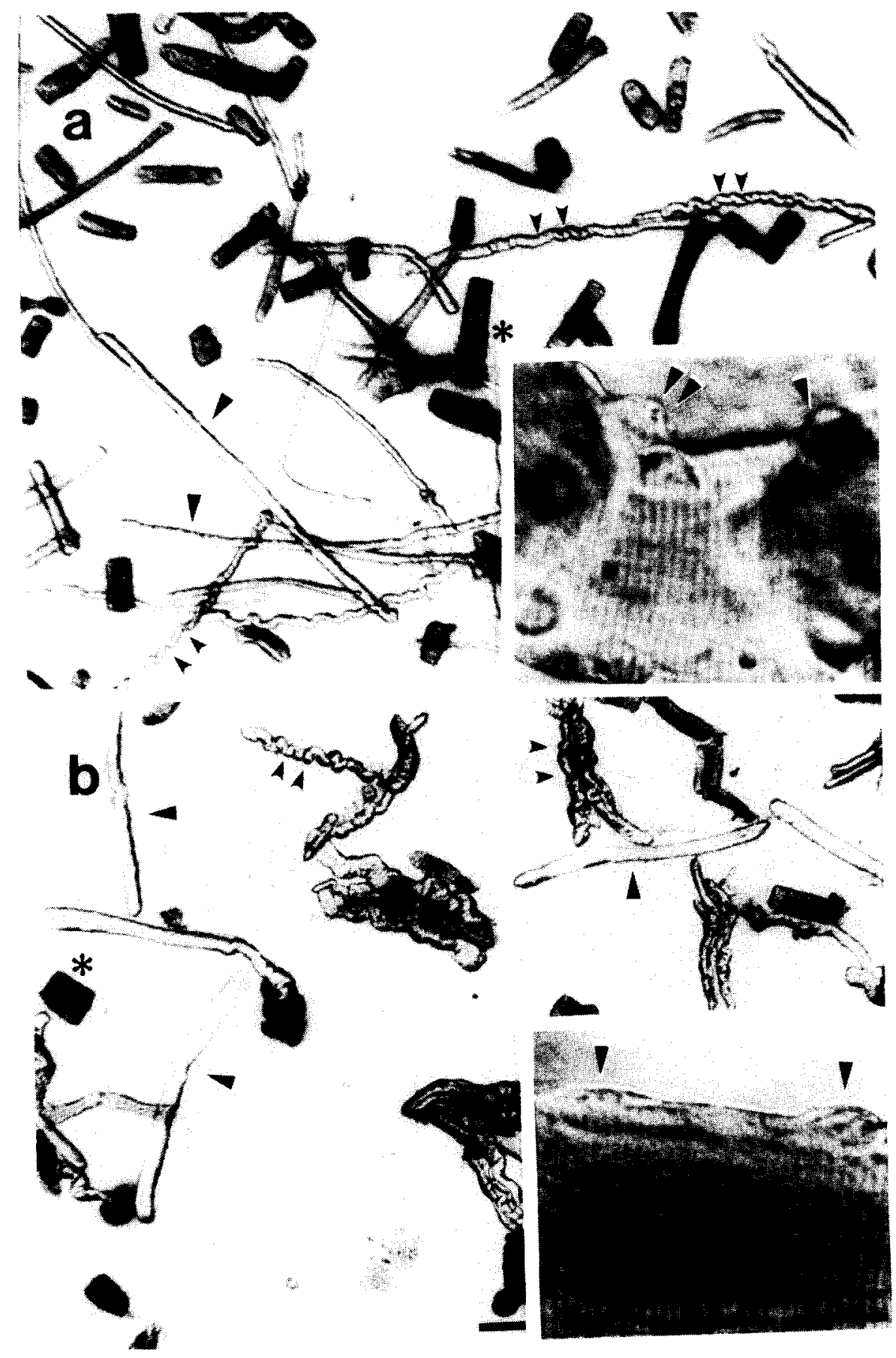



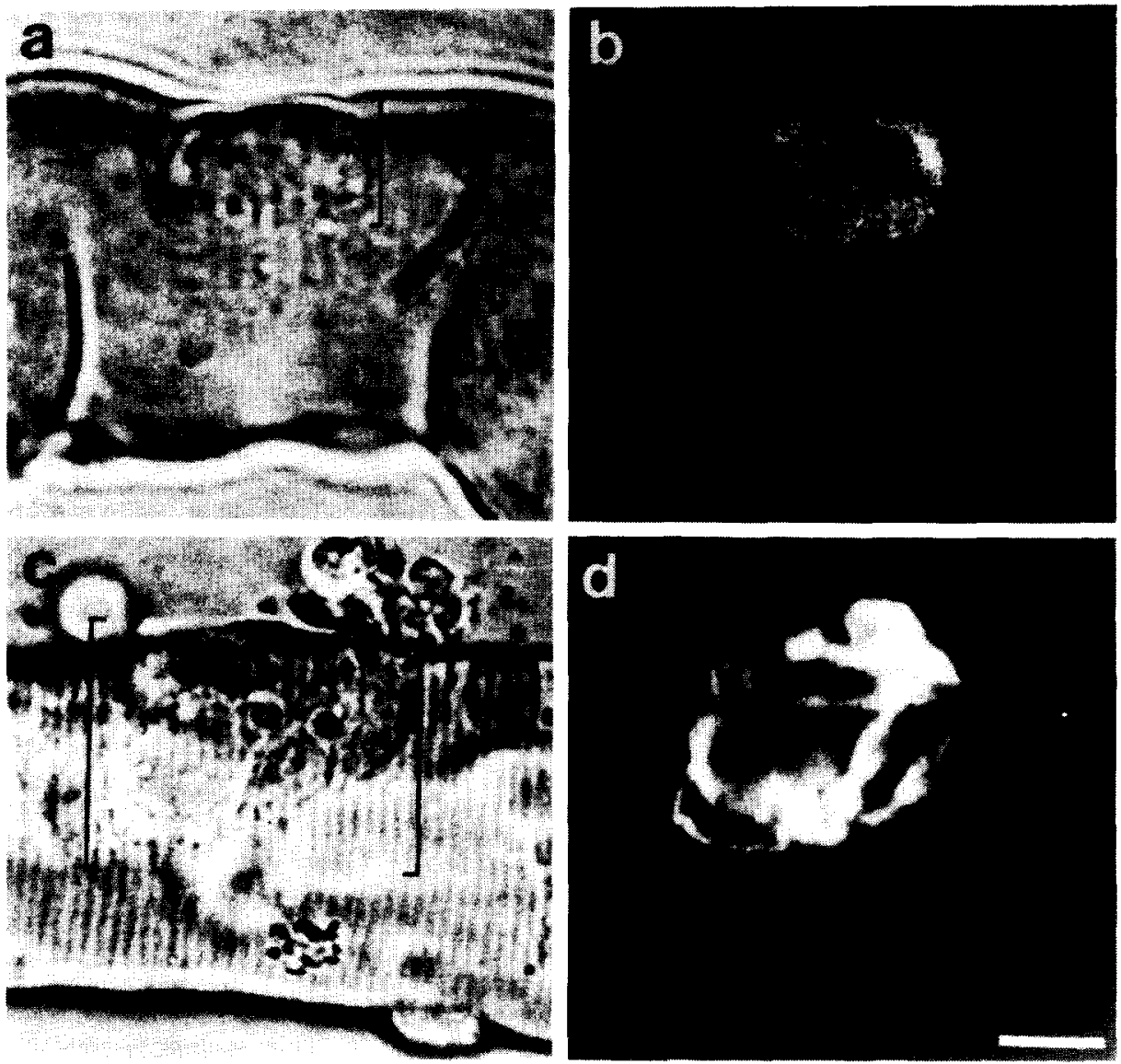

Fig. 2. Comparison of endplates on young adult and aged muscle fibers that were cultured for 1 day. (a) Phase contrast micrograph of young adult muscle fiber; the endplate is bracketed. (b) Fluorescence micrograph of the same fiber as in (a). R-BTX fluorescence was seen in the endplate region bracketed in (a). (c) Phase contrast micrograph of an aged muscle fiber; the endplate is bracketed. (d) Fluorescence micrograph of the same fiber as in (c). Note the detailed outlining of complex junctional folds revealed by R-BTX fluorescence and the clusters of nuclei at both young adult and aged endplates (brackets). Bar (a-d) represents $20 \mu \mathrm{m}$.

Fig. 1. Young adult and aged muscle fibers cultured for 1 day. (a) Young adult culture, prepared from the flexor digitorum brevis (FDB) muscles of an animal that was 5 months old. Some viable fibers that excluded trypan blue were contracted to some degree (double arrows); uncontracted fibers are indicated by single arrows. Dead fibers or fragments (*) did not exclude trypan blue. Inset: Phase contrast criteria for viability included peripheral location of myonuclei (arrow) and distinct striations. The endplate (double arrows) was seen as a raised mound. (b) Aged muscle culture, prepared from the FDB muscles of a 24-month-old animal. These cultures also contained non-viable fibers (*). In comparison to young adult cultures, viable aged fibers were more often found in clumps and more fibers were contracted (double arrows). Single fibers of the type monitored in these experiments, were not contracted (single arrows). Inset: Phase contrast criteria for viability were the same for young adult and aged fibers, i.e. peripheral nuclei (single arrows) and definite striations. Bars: (a and b), $200 \mu \mathrm{m}$; insets, $20 \mu \mathrm{m}$. 
aged muscle in vivo [29]. The individual cultured fibers also had AChE and AChRs co-localized at the junctions initially, as did the muscle fibers in vivo. (Endplates at later stages in culture are illustrated in Fig. 6 and discussed below).

\section{Fiber viability}

Viability of aged muscle fibers with time in culture was the same as that of young adult fibers (Fig. 3). Fewer aged fibers could be obtained in dissections of aged FDB muscles than in dissections of young adult fibers because of the presence of more fat and connective tissue in the FDB that prevented recovery of large numbers of single fibers (see above). Once in culture, however, no significant differences were observed in numbers of viable fibers in aged or young adult muscle preparations over a 10-day period. During the dissociation process, many fibers are broken and either die during preparation or subsequently die within the first day in culture Therefore by day 1 in culture, only approximately $60 \%$ of the fibers are viable (Fig. 3). However, there is little cell death in the ensuing 9 days in either young adult muscle cultures or those prepared from aged muscles.

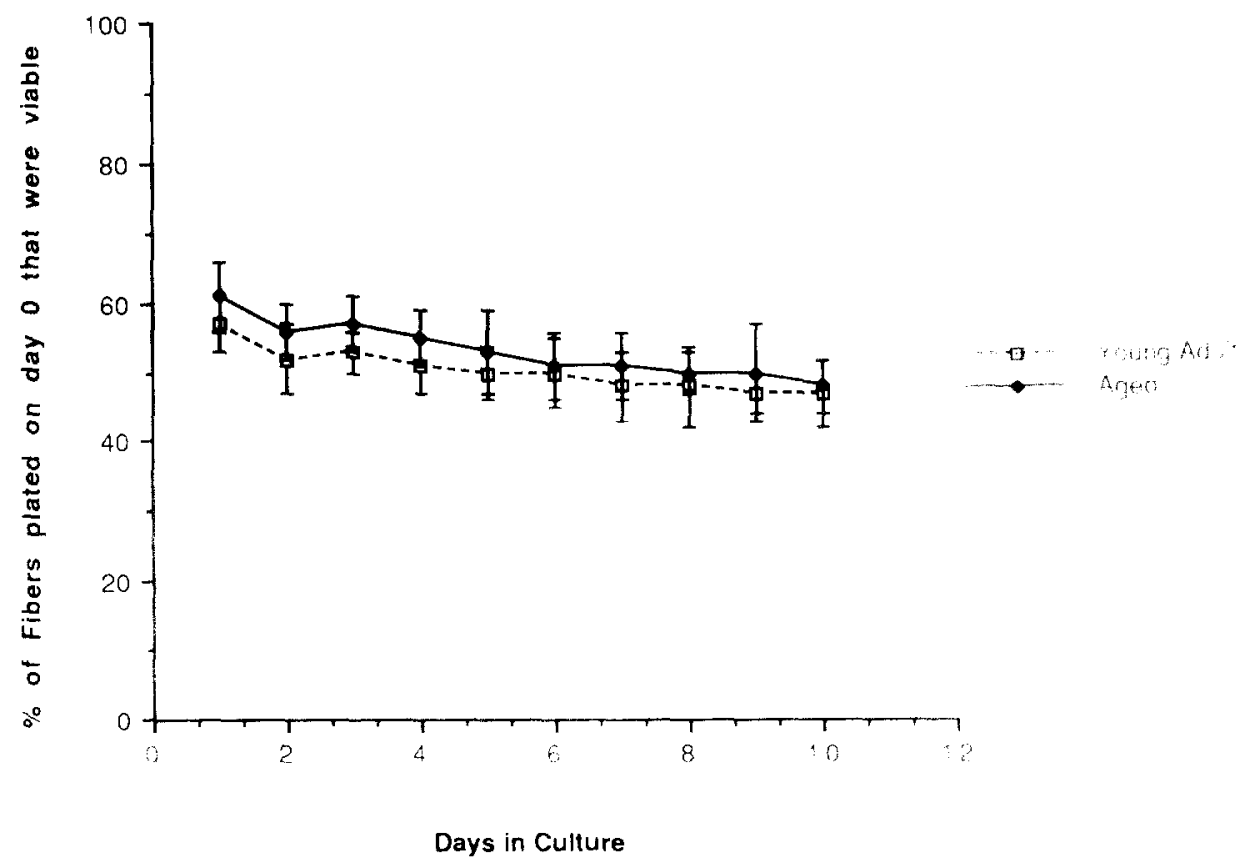

Fig. 3. Fiber viability in cultures of young adult and aged muscle fibers, with time in culture. When fiber are initially prepared for culture, $70 \%$ of the fibers plated on day 0 are viable. Approximately $10 \%$ moro fibers die during the next day in culture; however, after that time, few other fibers are lost in either young adult or aged muscle fiber cultures. Viability studies were done on cultures containing between 200 and 300 fibers/dish. The data are expressed as mean percent of all fibers in the dish \pm S.F.M.; $n=6$ sets cultures of each type. $-\square-$, Young adult; - - , aged. 


\section{Neurite contacts in vitro}

In order to study reinnervation of individual cultured muscle fibers and to search for molecules that might provide cues to reinnervating nerve, we added ventral spinal cord explants from embryonic rat to the muscle fiber cultures [19]. Adult spinal cord does not survive in tissue culture; therefore, embryonic spinal cord, which survives well and which is capable of profuse neurite outgrowth [19], was used. The neurites grew out and made large numbers of contacts with both aged and young adult isolated individual skeletal muscle fibers.

Within 2 days, in both kinds of cultures, neurites grew out around the entire perimeter of the explant, increased in length and number with time, and often grew beyond muscle fibers.

We had no difficulty recognizing the location of the former junctional regions on muscle fibers throughout the culture period, since we had recorded the stage micrometer coordinates of the original endplate location on day 1 in culture using the criteria described in the Methods section. However, this location was confirmed in the vast majority of cases by the presence of at least one molecular junctional marker and often by the presence of persisting junctional folds as well (Fig. 4).

During the first 5 days in culture, the number of contacts of spinal cord neurites with junctional and extrajunctional regions of young adult and aged muscle were similar (Fig. 5). However, by 7 days, significantly greater numbers of aged than young adult muscle fibers had neurite contacts in both the junctional and extrajunctional regions. At 10 days, the last time examined, there was still a significant difference between numbers of junctional contacts on aged and young adult fibers (Fig. 5).

By 10 days in culture, the number of extrajunctional contacts found on aged muscle fibers was far greater than that on young adult fibers: of 3785 total young adult fibers examined only 71 extrajunctional contacts were found. All had associated gelasmin; none had AChE or AChRs. In contrast, of 3519 total aged fibers examined, a total of 8644 extrajunctional contacts were found; all were associated with gelasmin. Of these, 77 had associated AChE and none had AChR.

\section{Is the number of neurite contacts correlated with neuron survival?}

Because the number of neurite contacts on aged muscle fibers might reflect the possibility that aged muscle fibers enhance the survival of embryonic neurons in culture, two types of experiments were performed.

We first measured the relative profusion of neurite outgrowth from ventral spinal cord explants and the length of neurites in cultures of aged and young adult muscle over time. We found that there was no significant difference in either of these measurements over the 10-day period. For example, on day 2 after the addition of spinal cord explants, neurites extended from the explant cores over the entire $360^{\circ}$ circumference in both aged muscle fiber cultures and those containing young adult fibers. Unfortunately it is not possible to determine the exact number of neurites 

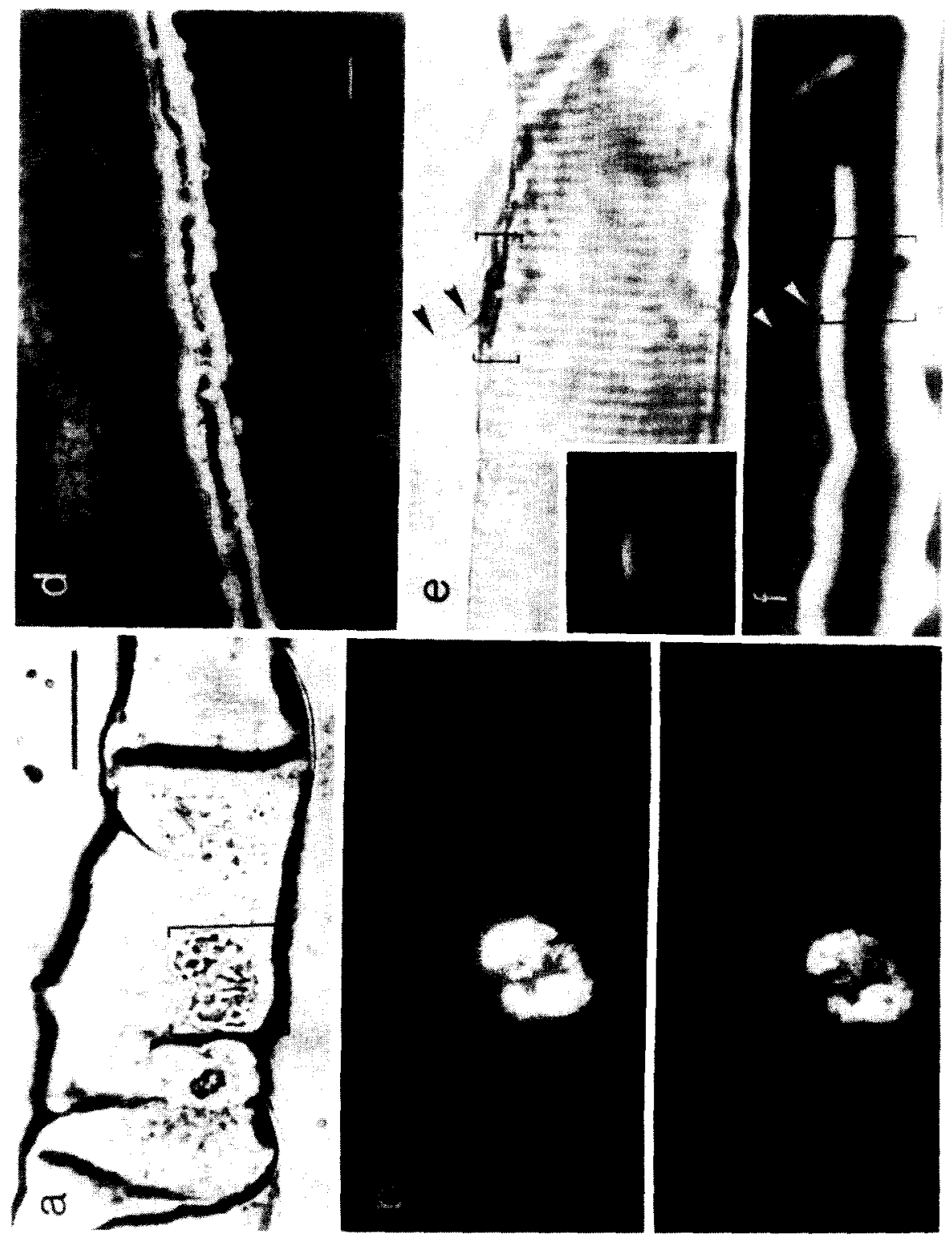


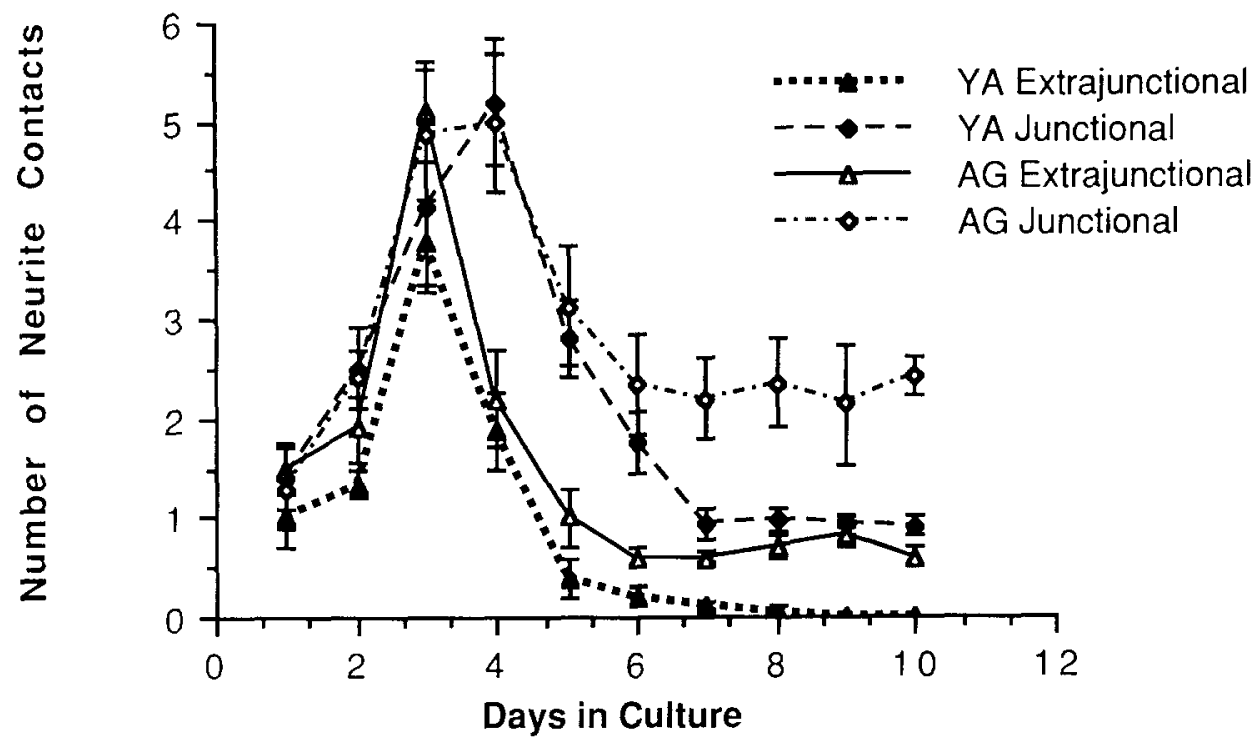

Fig. 5. Neurite contacts on aged and young adult individual muscle fibers in culture were counted as described in the Methods section. Total numbers of neurite contacts/unit area are graphed here. Note that the synaptic area is 1 unit in size. Aged fibers are those between 17-26 months old (595 fibers comprise this sample; at least 50 fibers were counted at each time point). Young adult fibers are those between 3-7 months old ( 655 fibers comprise this sample; at least 50 fibers were counted at each time point). The error bars represent the mean \pm S.E.M. for each sample.

Fig. 4. Gelasmin at the junctions of cultured muscle fibers from aged and young adult animals. Flexor digitorum brevis muscles of aged or young adult animals were isolated as described in the Methods section. (a) Muscle fiber from a 24-month-old animal in culture (day 1). The endplate is in brackets. (b) Fluorescence micrograph of the fiber shown in (a). The fibers were labeled with $0.5 \mu \mathrm{g} / \mathrm{ml}$ of SBL-2 the monoclonal antibody to gelasmin and a second step (1/1000) dilution of fluorescein conjugated rabbit anti-mouse IgG. After appropriate washes (detailed in Methods), the fiber was viewed with optics suitable for visualization of FITC. In (c) the same fiber was stained simultaneously with rhodamine-conjugated alpha-bungarotoxin (as described in Methods). The fiber was then viewed with optics suitable for RITC. Note that the distribution of gelasmin and R-BTX at the endplate is the same. In (d), a nerve explant (upper left out of focus) with many neurites radiating from it is seen in this culture of muscle fibers from a 20-month-old animal. Even after 8 days in culture, note the large number of extrajunctional contacts remaining on this fiber. Two are indicated with arrows. In (e) a young adult fiber 8 days in culture with a single persistent neurite contact (double arrow indicates the neurite) at the former junctional region is seen. The junctional region is in brackets in this phase contrast micrograph. Insert: gelasmin, identified with SBL-2 as in (b) is present at the endplate; none is present in the extrajunctional region of this fiber. (f) A neurite contact that persists in the extrajunctional region of an aged fiber after 8 days in culture is seen in brackets. Gelasmin is found along the path of the neurite (insert) as revealed by staining with SBL1 and a fluorescein-conjugated second step antibody as in (b). Note the exploring growth cone tip (lower right inside the bracket) does not have gelasmin associated with it. Scale bars: a,b,c,e,f, $20 \mu \mathrm{m} ; \mathrm{d}, 30 \mu \mathrm{m}$. 
(and therefore the density of neurites per unit area) because so many are in fascicle bundles. However, we observed no significant difference in the average length of the fascicle bundles on days 2 and 10 for young adult and aged muscle fiber cultures. day 2 YA: $932 \pm 23 \mu \mathrm{m}($ mean \pm S.E.M., $n=50)$; day 2 Aged: $908 \pm 41 \mu \mathrm{m}(n=$ 42). On day 10, neurites also extended over the entire circumference, but it was not possible to determine the density of neurite outgrowth, which was profuse. Again however, the average length of the neurites was similar. In young adult musck cultures, neurite length averaged $1.6 \mathrm{~mm} \pm 0.1 \mathrm{~mm}(n=35)$ and in aged muscle fiber cultures, neurite length averaged $1.5 \pm 0.2 \mathrm{~mm}(n=22)$.

\section{Survival of dissociated spinal cord neurons in aged and young adult nerve-muscte cultures}

Despite the fact that neurite length and density of outgrowth from spinal word explants appeared to be similar in young adult and aged muscle cultures, the experiments cited above do not answer the question of whether the aged musck fibers prolong the life of spinal cord neurons. Therefore, we dissociated ventrai spinal cord explants into single cells and plated $1 \times 10^{5}$ spinal cord cells/culture of young adult or aged muscle fibers. Three muscle fiber densities were selected for initial plating: 50 muscle fibers; 100 muscle fibers and 300 muscle fibers/culture of each type. The number of neurons surviving in the cultures was followed over a 10 day period. While greater numbers of neurons survived in the cultures containing the largest numbers of muscle fibers, there was no significant difference in the numbers of surviving neurons in the aged muscle fiber cultures a compared to the young adult muscle fiber cultures. For example, at day 10 , in the cultures that con tained 300 muscle fibers on day 1 , the number of surviving neurons in aged muscl cultures was $8.6 \pm 0.6 \times 10^{4}$ (S.E.M., $n=6$ ) and for young adul muscle cultures the number of surviving neurons was $8.9 \pm 0.7 \times 10^{4}$ (S.E.M., $n \cdots 6$ ).

\section{Gelasmin, AChE and AChR distribution over time}

In order to determine whether gelasmin is a good candidate as a molecula: mediator of neurite-muscle interactions, we next examined whether gelasmin was present at neurite contact sites on the cultured aged and young adult muscle fibers. We used the monoclonal antibody SBL-2, which is highly specific for gelasmin [12], and a fluorescent second step antibody to label fibers in the presence and absence of spinal cord explants (Fig. 4). In all cases examined, the (former) junctional region on the fibers was located by the stage micrometer coordinates and in most cases, could also be recognized by morphological and/or histochemical criteria. Fibers were observed with Smith interference contrast optics and cultures were stained for gelasmin and AChE activity which were usually still present on the muscle fibers The basal lamina-associated synaptic molecule AChE persists for very long periods of time (up to 11 months) on denervated skeletal muscle [31]. It persisted in the vast majority of fibers examined in these studies during the 10 day culture period (Figs 4 and 6). 
AChRs were also monitored. These molecules are usually very sensitive to denervation $[30,31,35,36]$. AChRs are usually located predominantly at the endplates of innervated muscle, but spread to extrajunctional regions quickly after denervation in the animal $[30,31,35,36]$. In the experiments described here, no attempt was made to monitor diffusely distributed receptors in extrajunctional regions.

Distribution of junctional molecules was the same on fresly isolated young adult and aged muscle fibers

We found $\mathrm{AChE}, \mathrm{AChRs}$ and gelasmin at virtually all endplates in samples of fresh muscle taken from animals 3-26 months old. Furthermore, $97 \%$ of muscle fibers in three freshly-isolated preparations of young adult muscle fibers ( $n=254$ total fibers counted) had AChRs co-localized with AChE and gelasmin at the synapse. In preparations of fresh aged muscle, all fibers examined had these molecules co-localized just prior to culture $(n=258)$.

Junctional molecules were distributed in a similar manner on young adult or aged muscle fibers at early stages in culture

Gelasmin, AChE and AChRs were found at the majority of contact sites (Fig. 6) on either young adult or aged muscle fibers in culture on days $1-4$ and similar numbers of young adult and aged fibers had co-localized gelasmin, AChE and AChRs in cultures of muscle alone or in nerve-muscle cultures (Fig. 6).

By day 5 in culture, and subsequently, many fewer young adult muscle fibers than aged muscle fibers retained junctional molecules; AChRs were absent most often

By 5 days in co-cultures of young adult fibers and spinal cord explants, however, only $47 \%$ of fibers maintained all 3 molecules at endplates (Fig. 6c). However, $91 \%$ of aged fibers did so (compare Figs. 6d and c). Significantly more fibers retained all three molecules in young muscle cultures without ventral spinal cord explants $(70 \%)$ (Fig. 6a). The difference in fibers retaining gelasmin was marked: $80 \%$ of young adult fibers in cultures without explants retained this molecule (Fig 6a); in co-cultures, only $55 \%$ of the fibers retained gelasmin at the endplate (Fig 6c). In contrast, gelasmin retention in aged muscle fiber cultures with and without spinal cord explants was the same (95\% and $94 \%$, respectively) (Figs $6 \mathrm{~b}$ and $6 \mathrm{~d}$ ). This was nearly double the number of fibers that retained gelasmin at the endplate in young adult nerve-muscle co-cultures.

By 10 days, gelasmin, $\mathrm{AChE}$ and $\mathrm{AChR}$ loss from neuromuscular junctions of young adult fibers both with and without spinal cord explants was far more pronounced than the loss of these molecules from aged fibers (compare Figs. 6a and $c$ with $6 \mathrm{~b}$ and d) and this was associated with a similar decline in the number of junctional connections on young adult fibers at this time (Figs. 6c and 6d). For example, double the number of aged fibers $(91 \%)$ had endplate gelasmin as 


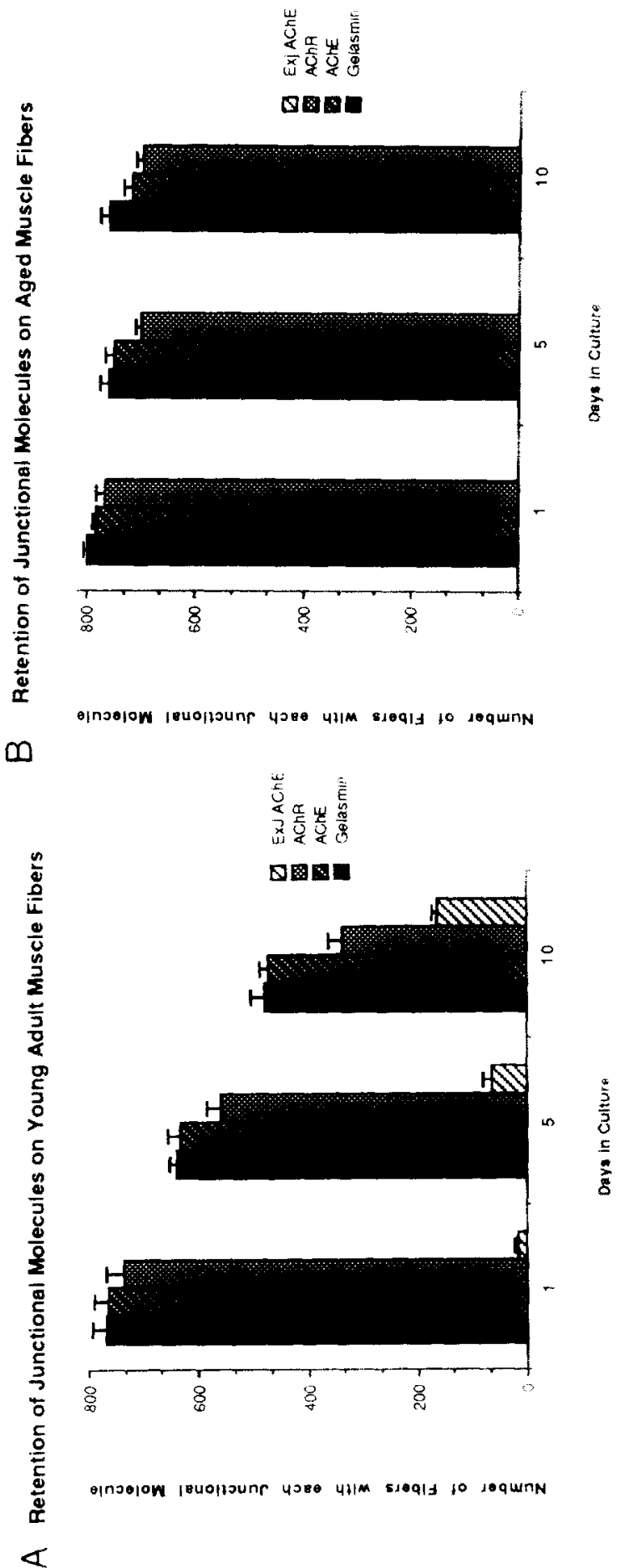




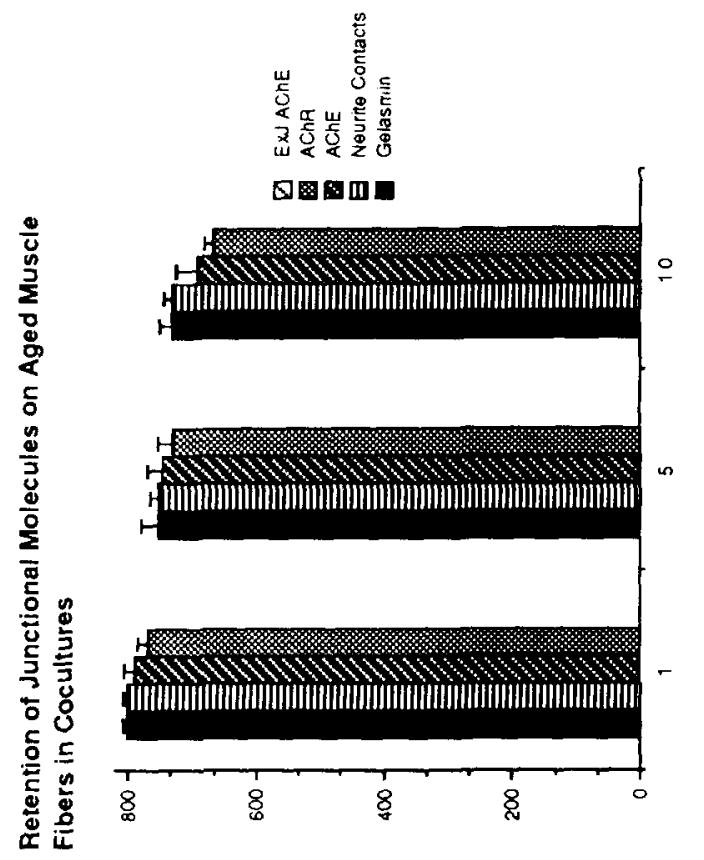

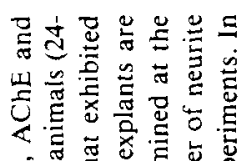

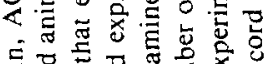

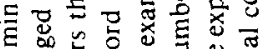

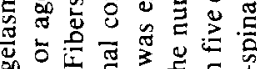

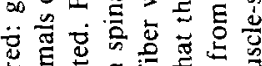

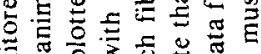

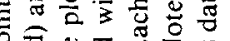

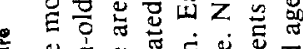

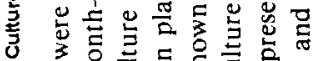

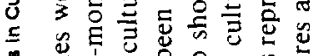

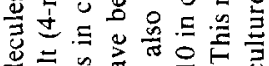

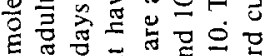

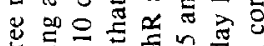

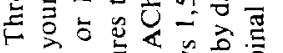

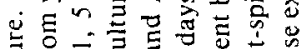

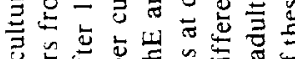

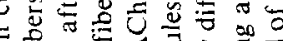

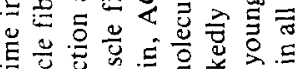

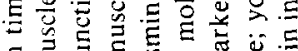

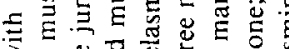

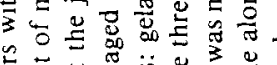

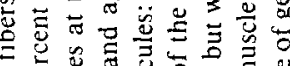

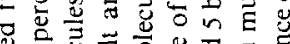

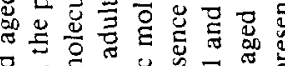

可

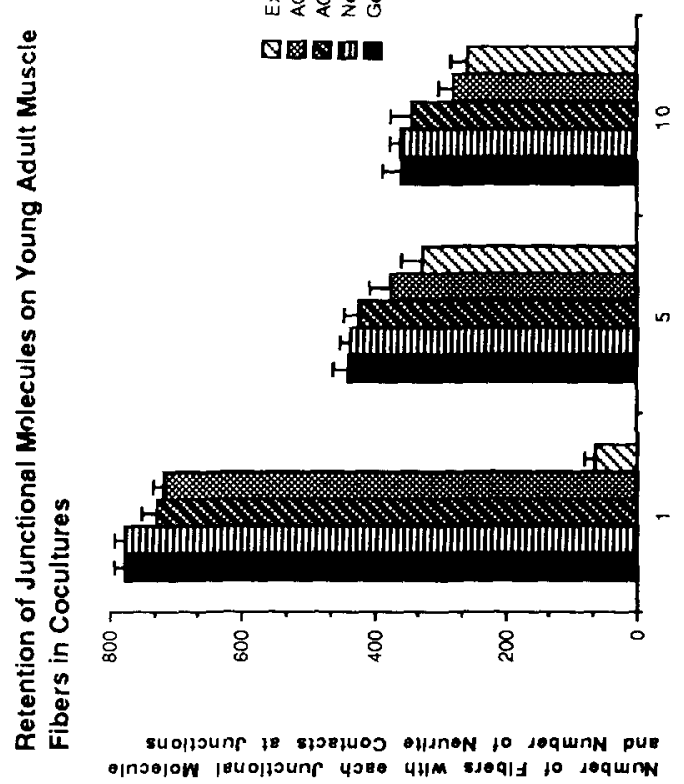

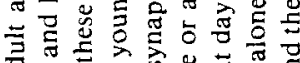

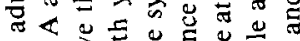

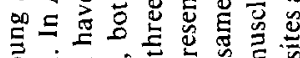

형 00

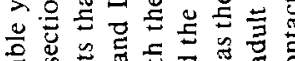

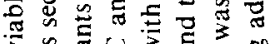

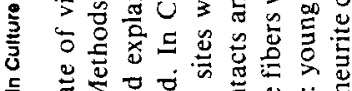

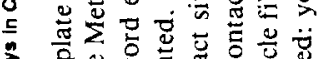

त्रे

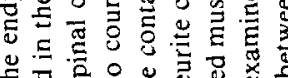

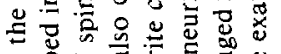

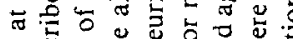

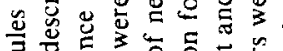

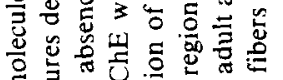

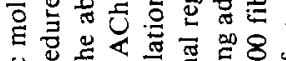

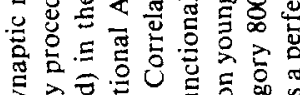

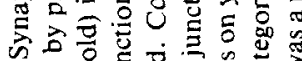

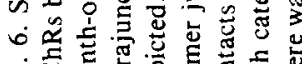

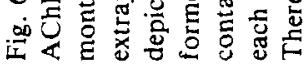


compared to young adult fibers $(45 \%$; Figs. $6 \mathrm{c}$ and $6 \mathrm{~d})$. The same was true of junc tional AChE ( $86 \%$ compared with $43 \%$; Figs. $6 \mathrm{c}$ and $6 \mathrm{~d}$ ).

Junctional $A C h R$ was lost more quickly than $\mathrm{AChE}$ in young adult muscle fiber cultures. In cultures of muscle alone, by day 10 in culture, $59 \%$ of fibers had junc tional AChE, but only $42 \%$ of the total fibers also had junctional AChR (Fig. 6a). In cultures with nerve explants, $43 \%$ of fibers had junctional AChE, but only $35 \%$ of the total fibers also had co-localized AChR (Fig. 6c). In cultures of aged muscle, $90 \%$ of the fibers in cultures of muscle fibers alone had both AChE and AChR (Fig. $6 \mathrm{~b}$ ) and $86 \%$ and $83 \%$ of fibers had $\mathrm{AChE}$ and $\mathrm{AChR}$, respectively, in nerve-muscle co-cultures (Fig. 6d).

Many young adult muscle fibers but very few aged fibers had extrajunctional AChE

Neurite contact and increased time in vitro were also correlated with an increase in the number of young adult fibers with extrajunctional AChE (Fig. 6c). Forty-one percent of the fibers in young adult nerve-muscle cultures had extrajunctional AChF: at day 5 as opposed to $9 \%$ of the fibers cultured without nerve (Fig. 6a). In cultures of aged muscle, however, no extrajunctional AChE was found in the presence or absence of neurites at this time (Figs. 6b and 6d).

Extrajunctional AChE did not appear on aged fibers in cultures of muscle fibers alone, but was observed on $32 \%$ of young adult muscle fibers at 10 days in cultures with explants (Fig. 6c). Of nearly 9000 neurite contact sites on aged fibers in nerve muscle co-cultures that we examined, 77 , or less than $1 \%$ had extrajunctional ACht (see above) at sites of nerve contact. As already stated, gelasmin was present at all of these contact sites.

Enhanced loss of junctional molecules appears to be correlated with neurite contact on young adult but not on aged muscle fibers in culture

Thus, aged fibers did not qualitatively lose AChE and AChR at the junction in response to nerve contact, but young adult muscle fibers did. We have since demonstrated that this loss occurs only on fibers contacted by nerve and not on other fibers in cultures that contain nerve explants that are not contacted by nerve (Barald and Jay, submitted).

\section{Correlation of neurite contacts in the junctional region and localization of gelasmin at the junction}

Gelasmin was always found at sites of nerve contact on all young adult and aged freshly isolated muscle fibers as well as cultured muscle fibers (Figs. 4 and 6). While gelasmin was present at virtually every site of nerve-muscle contact (Fig. 4) AChE was present less frequently and AChRs still less often (Fig. 6).

If one compares the distribution of neurite contacts, gelasmin. AChE and AChR at days 1,5 and 10 in culture (Fig. 6), both young adult and aged fibers received many neurite contacts by day 5 , but while the number of fibers with neurite contacts 
continued to increase in cultures of aged fibers, they decreased markedly in cultures of young adult muscle by day 10 . At all time points, however, neurite contact points had gelasmin, including the ectopic extrajunctional contacts that persisted on fibers after 5 days in culture (see above). The sole exception included 2 fibers in a single experiment done on a set of young adult cultures at day 10. The correlation was $100 \%$ in five other experiments (number of fibers examined in total, 800 aged and 800 young adult muscle fibers).

Thus, our study of aging muscle has helped to strengthen the correlation of gelasmin with neurite contacts, which we had already observed on young adult fibers [13]. Although aged fibers may regulate gelasmin or similar molecules differently from young adult fibers, and different patterns of nerve contact are seen in both junctional and extrajunctional regions of these fibers over the 10-day culture period, the presence of gelasmin is correlated with both junctional and extrajunctional neurite contact sites, while $\mathrm{AChE}$ is not.

\section{DISCUSSION}

We have developed a tissue culture system to serve as a model of vertebrate muscle reinnervation. We have used this experimentally manipulable system to show that steady state numbers of neurite contacts in the junctional and extrajunctional regions on both aged and young adult muscle fibers are correlated with the presence of a synapse-enriched molecule, gelasmin. By observing individual neurite contacts on a daily basis over the 10 days of this study, we believe that the neurite contacts persist at both young adult and aged junctions over this relatively long time in culture. During this time, muscle fiber viability in cultures of aged muscle was as good as that in cultures of young adult muscle and we made these observations on similar numbers of fibers in each age range.

In order to answer the question of whether the neurite contacts and synaptic molecules we have examined "persisted" at individual endplates throughout the culture period, we are presently using cinematography to follow the entire time course of nerve-muscle interactions.

Although all three junctional molecules examined (gelasmin, AChE and AChRs) were found on more aged than young adult muscle fibers over the 10-day culture period, only gelasmin, but not AChE or AChRs was present at essentially all sites of neurite contact regardless of the age of the animal from which the fibers were obtained or time in culture.

Our results suggest that the regulation of these three synaptic molecules (gelasmin, AChE and AChR) may differ in young adult muscle and aged muscle, although the mechanisms by which the three synaptic molecules might be retained (or replenished) on aged fibers are presently unknown. There are at least two possible explanations for the differences between the aged and young adult muscle fibers in synaptic molecule localization. (1) It is possible that aged junctions have greater 
amounts of all three of the synaptic molecules than do young adult fibers, reflecting the larger and more elaborate nature of these aged muscle junctions [6-8]. (2) Alternatively, the turnover time and/or stability of AChE, AChRs and gelasmin may be different in young adult and aged muscle fibers, and each of these molecules may be affected differently by the culture environment. Cytoskeletal elements that are involved in the anchorage of receptors at the junction [32], might also be more labile in young adult than in aged muscle fibers.

However, there are reports in the literature that the neuromuscular junctions of ageing soleus muscle undergo extensive remodelling in vivo [6-8,16,18]. We found no evidence of extensive remodelling or denervation of ageing FDB muscles in vivo (Barald and Jay, unpublished). In this respect the FDB may resemble the diaphragm, which also does not undergo extensive neuromuscular junctional remodelling [6-8]. This may reflect the use that the FDB muscles of aged animals receive in the animal, since muscles that receive constant use appear to undergo less junctional alteration and denervation than those muscles that are less used $\left[\begin{array}{ll}6 & -8\end{array}\right]$

Our observations of cultured aged and young adult rat muscle fibers agree with previous reports that $\mathrm{AChE}$ persists longer than acetylcholine receptors at original endplates in vivo [31,33]. In our study, this was particularly noticeable after day ? in culture. In the cat, junctional AChRs persisted for 6--7 months, but cholinesterase staining was found 11 months after denervation [33]. However, since we found that AChRs disappeared from a greater number of young adult endplates than aged end plates by 5 days in culture, molecular changes that occur with ageing may result in: the retention of AChRs at the endplate

In studies of cultured embryonic Xenopus muscle [34], Kuromi and Kidokoro found that if nerve-muscle contacts that formed in culture were allowed to persist for 3 days before they were severed by laser ablation of the neuron, $50 \%$ of the junctional AChRs became stabilized; if the nerve-muscle contacts were allowed to persist for only 1 day in culture however, $80-90 \%$ of the AChR clusters at the junction disappeared within $7 \mathrm{~h}$ after laser ablation of the nerve [34]. We have not examined the persistence of junctional $\mathrm{AChRs}$ after severing nerve-muscle contacts that formed in culture. We also did not attempt to observe diffusely distributed AChR; nor did we attempt to quantify the level of $A C h R$ in either junctional of extrajunctional regions.

Changes in the frequency of nerve sprouting $[7.8 .15]$ at aged neuromuscular junctions, as well as increased frequency of denervated junctions $[7,8]$ during the ageing process, may contribute to the "constant" remodeling of junctions seen in the ageing rat soleus muscle $[7,8]$. However, muscle types such as the predominant: slow soleus may respond in a highly individual (and different) manner to the ageing environment than do the predominantly fast diaphragm $[7,8]$ or predominantly fas: FDB. Changes during ageing at the soleus neuromuscular junction have, in fact. been found to be especially pronounced $[7,8]$. We do not find evidence of extensive neuromuscular junction remodelling or denervation of aged FDB muscles in wivi; 
(Jay and Barald, unpublished). However, if nerve-induced remodeling does occur in the FDB, it may affect the AChRs on aged FDB fibers appreciably more than it affects $A C h E$ or gelasmin distribution. We see no evidence in the culture system that aged FDB muscle fibers are in any way refractory to nerve contact, the first step in reinnervation.

We are presently examining nerve contacts electrophysiologically for evidence of miniature endplate potentials or other functional signs of innervation, since nerve contact alone, of course, does not prove that the fibers are functionally innervated.

Although the spread of extrajunctional acetylcholine sensitivity takes place 1 to 2 days after denervation of muscle in vivo [30,31,35,36], acetylcholine receptors were not seen in the extrajunctional region of aged fibers at early times in our cultures. However, we only noted the presence of receptor clusters; diffusely distributed receptors were not documented. The appearance of extrajunctional AChRs in other culture systems was found to be delayed until approximately 6 days after denervation [20]. Our observations agree with this report, in that extrajunctional AChRs also appeared on young adult but not aged fibers in this culture system at 5-6 days in culture (Jay and Barald, submitted). This could be due to a greater sensitivity of young adult fibers to nerve contact than aged fibers; however, the mechanisms will have to be addressed in future experiments.

Loss of junctional molecules could be due to release of urokinase-like proteinases or metalloproteinases from neurite growth cones as they pass over the surtace of the muscle fibers [37]. Neurites release a number of such proteinases [37-43] some of which, at least theoretically, have been implicated in the remodelling of the neuromuscular junction [43]. The proteinases, released locally by exploring growth cones, destroy the scaffolding that anchors synaptic molecules [43]. Synaptic molecules on young adult muscle fibers and/or the extracellular matrix on these fibers may be more sensitive to these proteinases than aged muscle. Alternatively, the aged muscle fibers may have appreciably more of the synaptic molecules initially at their larger and more elaborate junctions. Removal of some of the molecules by proteinase digestion may still leave enough behind to mediate nerve contact formation. However, since loss of junctional molecules occurred in cultures without nerve (only at a slightly slower rate), nerve-mediated losses may not be as important as environmental factors or intrinsic muscle properties in maintenance or loss of the molecules.

The most important finding of this study is that, after 5 days in culture, significantly fewer neurite contacts were found in the former junctional regions of young adult as opposed to aged muscle fibers, although gelasmin was present at both aged and young adult nerve-muscle contact sites. Gelasmin was found at all sites of nerve-muscle contact, even in the absence of detectable AChE or AChRs. Because gelasmin induces clustering of AChRs on embryonic myotubes $[11,12]$ its localization may serve as a focal point for AChR accumulation and/or stabilization. Furthermore, others have previously reported that agrin [44], a molecule to which 
gelasmin bears some limited resemblance, may organize AChE at the neuromuscular junction. We have not investigated such a role for gelasmin. Although gelasmin bears certain similarities to agrin, we have recently found that gelasmin but not agrin affects AChR alpha-subunit messenger RNA levels, and that gelasmin acts synergistically with calcitonin gene related peptide (CGRP) to do so (Barald et al submitted). Thus, the presence of gelasmin or some similar molecule may be a focal point for synaptic molecular organization.

Both gelasmin retention at endplates and numbers of neurite contacts in the junctional and extrajunctional regions of aged fibers differed greatly from those found on young adult fibers after 7 days in culture. This could reflect the amount of gelasmin present at the junction, which may be greater on aged than on young adult muscle, or may be due to some as yet unknown factor(s) or molecules.

Early in the culture period, large numbers of neurite contacts are found in both junctional and extrajunctional regions, but alter 10 days, while contacts in both regions decline, the contacts that remain are found exclusively in the junctional region on young adult fibers. This elimination of extrajunctional contacts and the reduction of contacts in the junctional region until they approach one contact site may be a recapitulation of innervation patterns seen during early synaptogenesis. Polyneuronal innervation (more than one neurite innervating a single junction) is seen on embryonic muscle fibers during development [45]; however, by the time synapses are stabilized, only one neurite contact per fiber remains. Initial contact by more than one neurite per fiber and an eventual reduction to a single neurite contact occurs during reinnervation of adult skeletal muscle [2] and on young adult fibers in culture also; extrajunctional contacts are eliminated in such cultures (Barald and Jay, submitted). However, in the case of the aged fibers, while elimination of both junctional and extrajunctional contacts occurs, more than one contact remains in junctional regions during the entire culture period and a diminution but not complete elimination of extrajunctional contacts is seen during the 10-day culture period. This could be due to the presence of increased amounts of gelasmin or of a yet undefined "synapse stabilization molecules" at both the junctional and extrajunctional neurite contact sites on aged fibers. Quantitation of such molecule: on individual muscle fibers will be difficult, but with ${ }^{3} \mathrm{H}$-labeled molecules and grain counting, might eventually be possible.

We cannot draw any causal inferences at this time between the larger number of neuromuscular contacts at aged junctions seen at 10 days and the persistence of the synaptic molecule gelasmin. We have not yet examined nerve-muscle contact points on cultured young adult or aged muscle fibers for more than the three synapse. related molecules reported here. Because many such "synapse-specific" or mors properly, synapse-enriched molecules exist $[5,10,11,12,32,43]$, we cannot rule out the possibility that another molecule acts as a cue for nerve to make and maintain contact with the fibers. However, because we have found a major difference between young adult and aged muscle fibers in the total number of fibers that retain 
gelasmin at late times, and gelasmin's presence is correlated with nerve-muscle contacts, it is a good candidate for a molecule that may mediate nerve-muscle contacts.

\section{ACKNOWLEDGEMENTS}

This research was sponsored by grants from NIH (NS17017, subproject), from the MDA, and from the Rackham Foundation to KFB and by a Rackham predoctoral fellowship and support from an NIH Developmental Biology training grant to JCJ. KFB was supported by a senior fellowship from the NIH NIA. We would like to thank B.M. Carlson, A.L. Dunathan, C. Carter-Su, T.J. Hinterberger and K.W. Tosney for many helpful discussions and for comments on the manuscript. S.L. Moskwiak, N. Smith, P. Jewett and L. Polacek contributed their skills to preparation of the typescript and figures. L.A. Polacek, E.C. Coucouvanis, F. Lin, M. Laus, M. Hillberg, and S. Kistka provided expert technical assistance; some of these students were supported by the Federal Work Study Program.

\section{REFERENCES}

1 M.C. Brown and W.G. Hopkins, Role of degenerating axon pathways in regeneration of mouse soleus motor axons. J. Physiol., (Lond.) 318 (1974) 515-545.

2 A. Gorio, G. Carmignoto, M. Finesso, P. Polato and M.G. Nunzi, Muscle reinnervation. II. Sprouting, synapse formation and repression. Neuroscience, 8 (1983) $403-416$.

3 E. Gutmann and J.Z. Young, The reinnervation of muscle after various periods of atrophy. $J$. Anat., 78 (1944) 15-57.

4 L.M. Marshall, J.R. Sanes and U.J. McMahan, Reinnervation of original synaptic sites on muscle fiber basement membrane after disruption of the muscle cells. Proc. Natl. Acad. Sci. USA, 74 (1977) 3073-3077.

5 J.R. Sanes and J. Couvault, Axon guidance during reinnervation of skeletal muscle. Trends in Neurosci., 8 (1985) $523-528$.

6 C.A. Cardasis and D.W. LaFontaine, Ageing rat neuromuscular junctions: a morphometric study of cholinesterase-stained whole mounts and ultrastructure. Muscle Nerve, 10 (1987) 200-213.

7 C.A. Cardasis and H.A. Padykula, Ultrastructural evidence indicating reorganization at the neuromuscular junction in the normal rat soleus muscle. Anat. Rec., 200 (1981) 41-59.

8 C.A. Cardasis, Ultrastructural evidence of continued reorganization at the ageing ( $11-26$ months) rat soleus neuromuscular junction. Anat. Rec., 207 (1983) 339-415.

9 J.R. Sanes, L.M. Marshall and U.J. McMahan, Reinnervation of muscle fiber basal lamina alter removal of muscle fibers. J. Cell Biol., 78 (1978) 176-198.

10 L.L. Rubin and U.J. McMahan, Regeneration of the neuromuscular junction: Steps toward defining the molecular basis of the interaction between nerve and muscle. In D.L. Schotland (ed.), Disorders of the Motor Unit, John Wiley, New York, 1982, pp. 187-196.

11 K.F. Barald, G.D. Phillips, J.C. Jay and I.F. Mizukami, A component in mammalian muscle synaptic basal lamina induces clustering of acetylcholine receptors. Prog. Brain Res. 7I (1987) 397408.

12 K.F. Barald, G.D. Phillips and J.C. Jay, Gelasmin: An acetylcholine receptor clustering factor derived from mammalian muscle extracellular matrix: I. Location on embryonic rat myotubes and at adult synapses. Development, in press.

13 J. Jay and K.F. Barald, Denervated single myofibers: Neurite interactions and synaptic molecules. Muscle Nerve, in press. 
$14 \mathrm{~J}$. Courtney and J.H. Steinbach, Age changes in neuromuscular junction morphology and acetylcholine receptor distribution in rat soleus muscle fibers. J. Physiol. (Lond). 320 (1981) 435 -..447.

15 D.O. Smith and J.L. Rosenheimer, Decreased sprouting and degeneration of nerve terminals of active muscles in aged rats. $J$. Neurophysiol., 48 (1982) 100-109.

16 M.A. Fahim and N. Robbins, Ultrastructural studies of young and old mouse neuromuscular junc. tions. J. Neurocytol., 11 (1982) 641-656.

17 J.L. Rosenheimer and D.O. Smith, Changes in plasticity at the neuromuscular junction during ageing. Proc. Int. Union Physiol. Sci., XV (1983) 448-456.

18 D.O. Smith and J.L. Rosenheimer: Ageing at the neuromuscular junction. In J.E. Johnson, It (ed.), Ageing and Cell Structure, Vol. 2, New York, Plenum Press, 1984, pp. 113-139.

19 J. Jay and K.F. Barald, Improved culture of individual muscle fibers with and without spinal cord explants in a collagen gel. J. Neurosci. Methods, 15 (1985) 229-234.

20 A. Bekoff and J.W. Betz, Properties of isolated adult rat muscle fibers maintained in tissue culture. J. Physiol. (Lond), 27I (1977) 537-547.

21 E.E. Goldman, Die aussere und innere Sekretion des gesunden organismus im lichte der vitalen farbung. Beitr. Klin. Chir., 64 (1909) 192-265.

22 B.B. Mishell and S.M. Shiigi. Selected Methods in Cellular Immunology, W.H. Freeman, Sar Francisco, CA, (1980), pp. 16-17.

23 D.R. Parks, V.M. Bryan, V.T. Oi and L.A. Herzenberg, Antigen-specific identification and cloning of hybridiomas with a fluorescence-activated cell sorter. Proc. Natl. Acad. Sci. USA, 76 (1979) 1962 $-1969$

24 K. Barald and D.K. Berg, High affinity choline uptake by spinal cord neurons in dissociated cell culture. Develop. Biol., 65 (1978) 90-99

$25 \mathrm{P}$. Ravdin and D. Axelrod, Fluorescent tetramethyl rhodamine derivatives of alpha-bungarotoxin preparation, separation, and characterization. Anal. Biochem., 80 (1977) 585-592, and correction. 83 (1977) 336

26 F. Moody-Corbett and M.W. Cohen, Localization of cholinesterase at sites of high acetylcholine receptor density on embryonic amphibian muscle cells cultured without nerve. $J$. Neurosci., $/$ (1981) $596-605$

27 S. Siegel, Non-parametric Statistics, McGraw Hill Book Co., New York, NY, 1956, pp. $184 \cdots 194$.

28 R. Bischoff, Proliferation of muscle satellite cells on intact myofibers in culture. Develop. Biol, , $/ 15$ (1986) 129-139.

29 M.A. Fahim, J.A. Holley and N. Robbins, Scanning and light microscopic study of age changes at if neuromuscular junction in the mouse. $J$. Neurocytol., 12 (1983) 13--25

$30 \mathrm{~J}$. Axelsson and S. Thesleff, A study of supersensitivity in denervated mammalian skeletal muscle J. Physiol. (Lond.), 147 (1959) 178-193.

31 D.M. Fambrough, Control of acetylcholine receptors in skeletal muscle. Physiol. Rev. 59 (1979) $165-227$.

32 S. Burden, Identification of an intracellular postsynaptic antigen at the frog neuromuscular junction. J. Cell. Biol., 94 (1982) 521-530.

33 J.H. Steinbach, Neuromuscular junctions and alpha-bungarotoxin-binding sites in denervated and contralateral cat skeletal muscles. J. Physiol. (Lond.). 313 (1981) 513-528

$34 \mathrm{H}$. Kuromi and Y. Kidokoro, Denervation disperses acetylcholine receptor clusters at the neuromuscular junction in Xenopus cultures. Develop. Biol., 104 (1984) $421-427$.

35 C.R. Slater and E.G. Allen, Acetylcholine receptor distribution on regenerating mammalian muscle fibers at sites of mature and developing nerve-muscle junctions. $J$. Physiol. (Paris). 80 (1985) 238 -246.

36 R. Miledi, The acetylcholine sensitivity of frog muscle fibres after complete or partial denervation J. Physiol. (Lond.), 147 (1960) 178-193.

37 R.N. Pittman, Release of plasminogen activator and a calcium-dependent metalloprotease $1 \mathrm{rom}$ cultured sympathetic and sensory neurons. Develop. Biol., 110(1985) 91 -.. 101.

38 D.A. Baxter, D. Johnston and W.J. Strittmatter, Protease inhibitors implicate metallendoprotease in synaptic transmission at the mammalian neuromuscular junction. ProC. Natl. Acad. Sci. USA, 80 (1983) $4174-4178$. 
39 A. Krystosek and N.W. Seeds, Plasminogen activator release at the neuronal growth cone. Science, 213 (1981a) $1532-1534$.

40 A. Krystosek and N.W. Seeds, Plasminogen activator secretion by granule neurons in cultures of developing cerebellum. Proc. Natl. Acad. Sci. USA, 78 (1981b) 7810-7814.

41 A. Krystosek and N.W. Seeds, Peripheral neurons and Schwann cells secrete plasminogen activator. J. Cell Biol., 98 (1984) 773-776.

42 G. Moonen, M.P. Grau-Wagemans and I. Selak, Plasminogen activator-plasmin system and neuronal migration. Nature, 298 (1982), 753-755.

43 M.J. Anderson and L.E. Swenarchuk, Nerve induced remodeling of basal lamina during formation of the neuromuscular junction in cell culture. Prog. Brain Res., 71 (1987) 409-421.

44 B.G. Wallace, R.M. Nitkin, N.E. Reist, J.R. Fallon, N.N. Moayeri and U.J. McMahan, Aggregates of acetylcholinesterase induced by acetylcholine receptor-aggregating factor. Nature (Lond.), 315 (1985) 574-577.

45 M.R. Bennett and A.G. Pettigrew, The formation of synapses in striated muscle during development. J. Physiol. (Lond.), 241 (1974) 515-545. 